\title{
Joint Network Coding for Interfering Wireless Multicast Networks
}

\author{
Jalaluddin Qureshi, Chuan Heng Foh and Jianfei Cai \\ School of Computer Engineering \\ Nanyang Technological University, Singapore \\ jala0001@e.ntu.edu.sg
}

\begin{abstract}
Interference in wireless networks is one of the key-capacity limiting factor. The multicast capacity of an adhoc wireless network decreases with an increasing number of transmitting and/or receiving nodes within a fixed area. Digital Network Coding (DNC) has been shown to improve the multicast capacity of non-interfering wireless network. However recently proposed Physical-layer Network Coding (PNC) and Analog Network Coding (ANC) has shown that it is possible to decode an unknown packet from the collision of two packet, when one of the colliding packet is known a priori. Taking advantage of such collision decoding scheme, in this paper we propose a Joint Network Coding based Cooperative Retransmission (JNCCR) scheme, where we show that ANC along with DNC can offer a much higher retransmission gain than that attainable through either ANC, DNC or Automatic Repeat reQuest (ARQ) based retransmission. This scheme can be applied for two wireless multicast groups interfering with each other. Because of the broadcast nature of the wireless transmission, receivers of different multicast group can opportunistically listen and cache packets from the interfering transmitter. These cached packets, along with the packets the receiver receives from its transmitter can then be used for decoding the JNC packet. We validate the higher retransmission gain performance of JNC with an optimal DNC scheme, using simulation.
\end{abstract}

\section{INTRODUCTION}

Wireless multicasting is seen as a bandwidth efficient mean of disseminating common information to multiple receivers. Various emerging applications such as wireless multi-player gaming [1] and multimedia broadcast (currently being standardised by the IEEE 802.11aa working group) are based on wireless multicasting. While a previous empirical study [2] on the deployment of wireless Access Points (AP) in metropolitan areas has shown that APs are often deployed in a chaotic manner, with several of these APs therefore often competing with each other for access to the same transmission channel. Therefore with an increase in wireless mutlicast data traffic, and an increasing density of wireless nodes within a fixed area competing for the same channel, an efficient solution is needed which addresses both the increasing wireless multicast bandwidth demand and the constraint of wireless interference.

Digital network coding (DNC) has been shown to be one such technique which improve the capacity of multicast wireless network [3], and its reliability gain [4] [5] for a non-interfering network. In DNC, multiple packets are coded together over Galois Field $G F(q)$, where $q$ is the field size. If the coding vector is randomly selected from the Galois Field, then such DNC scheme is known as Random Linear
Network Coding (RLNC) [1] scheme. If this coding vector is selected deterministically then such DNC scheme is known as deterministic network coding, the most commonly used form of the deterministic network coding is known as XORcoding [4] [5], i.e. deterministic network coding over $G F(2)$.

Recent works have shown thats network coding can also be performed at the physical layer. Such network coding performed at physical layer, known as Physical-layer Network Coding (PNC) [6] and Analog Network Coding (ANC) [7] can improve the throughput order for multi-pair unicast transmission in ad-hoc wireless network [8]. In PNC/ANC, a node can decode the unknown packet $c_{2}$ from the collided packet $c_{1} \odot c_{2}{ }^{1}$, provided that the node has packet $c_{1}$ a priori. The key difference between PNC and ANC is that, PNC requires the colliding packets to collide in a perfectly synchronised manner, whereas in ANC, the colliding packet need not necessarily collide perfectly synchronised, which therefore makes ANC a more practical coding scheme for implementation. Therefore in our implementation we use the ANC scheme.

While a significant amount of work has been done to characterise the throughput benefits of DNC [3] [4] and ANC [8] [9] in isolation, to the best of our knowledge there has been no work done so far which characterises the joint benefits of DNC and ANC. Further, so far, ANC applications has only been limited for simple relay networks. Therefore our current work is also the first work of its kind extending the application of ANC beyond relay networks.

In this paper we demonstrate the throughput gain by jointly using ANC and DNC (deterministic XOR-coding) to transmit packets to receivers in a single-hop setting where two wireless multicast group interfere with each other, which we call Joint Network Coding using Cooperative Retransmission (JNC-CR). The rest of the paper is organsied as follow. We present an overview of related works in Section II. In Section III, we characterise the system model of the network. We then present the JNC-CR protocol design, along with an illustrating example in Section IV, followed by simulation results in Section V and summary of our work in Section VI.

\section{RELATED WORK}

Our current work is primarily an extension of our previous work, Cooperative Retransmission (CR) through collission [9].

\footnotetext{
${ }^{1}$ We use the $\odot$ notation to denote the collision operation, and $\oplus$ to denote XOR-coding operation.
} 


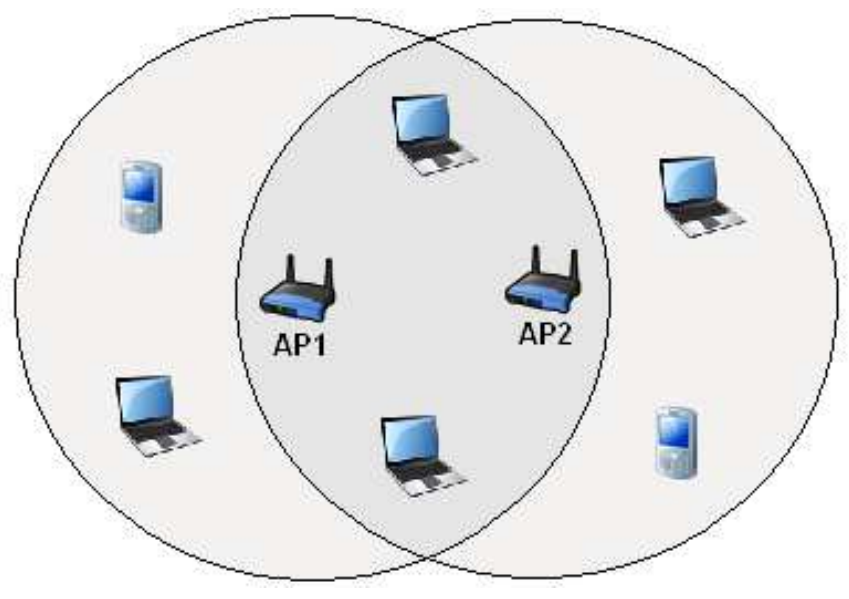

Fig. 1. Two interfering multicast network, with $\mathrm{N}=3$ and $\mathrm{M}=1$.

While in our previous work we had shown the retransmission gains of utilising ANC over IEEE 802.11 ARQ (Automatic Repeat Request) for 2 unicast transmissions interfering with each other, in our current work, we demonstrate the retransmission gains of JNC-CR over an optimal DNC scheme for 2 multicast transmissions interfering with each other.

\section{A. DNC Retransmission scheme}

A bulk of previous works [1] [4] [5] [10] [14] on network coding based retransmission schemes have been limited towards using DNC, comparing the performance gain of DNC over ARQ retransmission schemes. Advantages in such coding gains come from the independent Bernoulli packet loss model in wireless networks, as experimentally shown [11]. In [11] the authors showed that while the average packet loss probability of 2 co-located identical receivers are similar, the receivers' packet loss burstiness may however differ under similar conditions. Hence, instantaneous packet loss for 2 such receivers receiving transmission from the same transmitter show no correlation pattern. Therefore for a transmitter $A P_{1}$ multicasting packet $c_{1}$ and $c_{2}$ to receivers $R_{1}$ and $R_{2}, c_{1}$ may be received by $R_{1}$ only while $c_{2}$ may be received by $R_{2}$ only, however unlike ARQ retransmission scheme where both the packets are retransmitted separately in 2 different time slots, in a DNC based retransmission scheme the packets $c_{1}$ and $c_{2}$ are XOR-coded as $c_{1} \oplus c_{2}$ and retransmitted in 1 time slot. Upon reception of this coded packet both $R_{1}$ and $R_{2}$ can decode the coded packet using the packet they had received earlier. Therefore DNC reduces the time slots needed to retransmit the lost packets from 2 time slots to 1 time slots for this example. Our previous work, CR [9] was the first work of its kind to improve retransmission gain using ANC rather than DNC. CR is implemented by two interfering APs using the common superimposed acknowledgement information, transmitted by the receivers in the interference region.

\section{B. Superimposed Acknowledgement}

Superimposed acknowledgement [12] [13] is an ACKthinning scheme for multicast transmission. In a superimposed acknowledgement each receiver in the multicast network transmits a unique ACK packet, which is embedded with a unique predefined bitstream patterns, such that the simultaneous collision of these packets will result in a different collided packet for different permutation of ACK packets colliding together. Therefore all receivers which have received the packet transmit their ACK packet in the same time slot. This collided ACK packet can then be used to determine which set of receivers have received the data packet. Because of the broadcast nature of these collided ACK packet by receivers in the interference region, both the APs have the packet reception status of these receivers.

\section{Cooperative Retransmission}

In a CR [9] scheme, 2 interfering APs retransmit selected lost packets simultaneously, resulting in a collided packet, which is then decoded by the receivers using ANC and packets opportunistically overheard from the interfering AP. Such cooperative retransmission scheme is implemented without any complex handshaking or scheduling procedure. Consider, for example unicast transmissions $A P_{1} \sim R_{1}$ and $A P_{2} \sim R_{2}$, interferring with each other and $R_{1}$ and $R_{2}$ located such that both the receivers can hear transmission from $A P_{1}$ and $A P_{2}$. Because of the broadcast nature of wireless transmission, it is therefore possible that $R_{1}$ may overhear (also known as opportunistic listening) packet destined for $R_{2}$ and viceversa. We use superimposed acknowledgement in such a CR scheme, wherein both $R_{1}$ and $R_{2}$ simultaneously transmit ACK packet for transmission received by both $A P_{1}$ and $A P_{2}$. Therefore both the APs are aware of packet reception status of $R_{1}$ and $R_{2}$. In such a network packet $c_{1}$ transmitted by $A P_{1}$ destined for $R_{1}$ can be overheard by $R_{2}$ but not by $R_{1}$, whereas packet $c_{2}$ transmitted by $A P_{2}$ for $R_{2}$ can be overheard by $R_{1}$ but not by $R_{2}$. Because of the superimposed acknowledgement scheme, $R_{2}$ will transmit ACK packet for $c_{1}$, and $R_{1}$ will transmit ACK packet for $c_{2} . A P_{1}$ and $A P_{2}$ can then simultaneously retransmit $c_{1}$ and $c_{2}$ in the same time slot which results in the collided packet $c_{1} \odot c_{2}$. Each of the receiver can then use the previously, opportunistically received packet to decode $c_{1} \odot c_{2}$ using ANC. This therefore reduces the total number of retransmissions needed from 2 to 1 for this example. In this work we further expand on the benefits of both ANC and DNC, and show that using JNC-CR offers a much higher retransmission gain.

\section{SySTEM MODEL}

Consider two APs, $A P_{1}$ and $A P_{2}$ multicasting packets to receivers in their network. Let $d_{A P}$ denote the distance between the two APs, and $r_{t}$ denote the transmission range of each AP, characterized as an omnidirectional radio propagation, with both the APs following the uniform power assignment scheme, i.e. transmitting at an equal transmission power, and at the same transmission rate. Consider that the interfering APs are overlapped such that $d_{A P}<2 r_{t}$. Each AP associates with $N$ client stations, which are uniformly distributed within the transmission range of the AP. Average packet loss probability 
$p_{i j}$ for transmissions from $A P_{i}$ to receiver $R_{j}$ follows an independent Bernoulli packet loss model [11], where $1 \leq i \leq 2$ and $1 \leq j \leq 2 N$. Receivers $1 \leq j \leq N$ are connected to $A P_{1}$ and receivers $N<j \leq 2 N$ are connected to $A P_{2}$. The total number of nodes in the overlap region is given as $2 M$, where $1 \leq M \leq N$. We assume uniform packet loss $p$ for both the network, such that $p_{i j}=p, \forall i, j$. The probability that a receiver in the non-interference region correctly receives a packet is given as $(1-p)$, whereas the probability that a receiver in the interference region correctly receives a collided packet is given as $(1-p)^{2}$ [9], as both the colliding packet need to reach correctly at the receiver. Packet batch size for transmissions by $A P_{i}$ is denoted as $B_{i}$, for simplicity we assume that $B_{1}=B_{2}=B$. For multimedia applications such as video streaming and file sharing, $B$ is usually a large value. We assume a reliable feedback mechanism, this is consistent with the previous assumptions used in similar works [4] [5] [10]. Further we also assume a reliable superimposed acknowledgement feedback mechanism in our protocol design, consistent with our previous work [9].

\section{A. Performance overview}

In our performance evaluation, we compare JNC-CR with an optimal DNC scheme. However since it is a NP-hard problem to decide whether an optimal number of transmission can be achieved for a given field $G F(q)$ [14], we therefore assume an infinitely large value of $q$ for DNC. Without loss of ambiguity we ignore the large coding overhead of $B \log _{2}(q)$ bits for DNC corresponding to an infinitely large value of $q$. The expected number of transmissions (and hence retransmissions) needed to transmit $B$ packets to $N$ receivers using DNC has been calculated in Equation 13 of [15]. We therefore use result from [15], with $q=\infty$ as a lower bound for DNC, for specified values of $B$ and $N$.

Packet overhead of a XOR coded packet is given as $N \log _{2}(q)$, where $q=2$. Implementing ANC has a total packet overhead of 128 bits [7]. Therefore the total packet overhead of a JNC packet is given as $(128+2 N)$ bits.

\section{JNC CR PRotocol DESIGN}

In JNC, packets are encoded at two level. In the first instance, each $A P$ XOR selected data packets bit-by-bit, and then both the APs simultaneously transmit the XOR coded packet, which results in ANC of the XOR coded packets. When a receiver receives a JNC packet, it first performs ANC decoding on the JNC packet to retrieve the XOR-coded packets. Once a receiver decodes the collided packet it then retrieves the XOR-coded packet, on which it then performs XOR decoding to retrieve the data packets.

The APs make XOR coding decision using BENEFIT coding algorithm [5], and ANC coding decision using a simple ANC-CR coding decision as shown in Table II. BENEFIT is a memory based heuristic coding algorithm which makes coding decision such that every receiver receives an innovate packet on the reception of the coded packet. An innovative packet, is a packet which the receiver can not generate using the set of packets it already has.

\section{A. Illustrating example}

TABLE I

TRANSMISSION MATRIX EXAMPLE

\begin{tabular}{|c|c|c|c|c|}
\hline & $c_{1}$ & $c_{2}$ & $c_{3}$ & $c_{4}$ \\
\hline$R_{1}$ & 1 & 0 & 0 & 0 \\
\hline$R_{2}$ & 0 & 1 & - & - \\
\hline$R_{3}$ & 0 & 0 & 0 & 1 \\
\hline$R_{4}$ & - & - & 1 & 0 \\
\hline
\end{tabular}

Consider for illustration a simple example where $A P_{1}$ is multicasting packets $c_{1}$ and $c_{2}$ to $R_{1}$ and $R_{2}$, while $A P_{2}$ is multicasting packets $c_{3}$ and $c_{4}$ to $R_{3}$ and $R_{4} \cdot R_{1}$ and $R_{3}$ are located in the interference region, whereas $R_{2}$ and $R_{4}$ are located in the non-interference region. The reception status of each packet is given in Table I, where ' 1 ' represents that the packet has not been received by the corresponding receiver, ' 0 ' represents that the packet has been received, while '-' denotes that the receiver is not within the transmission range of the AP transmitting that packet. In an ARQ based retransmission scheme, $A P_{1}$ and $A P_{2}$ will retransmit these lost packets in different time slots, therefore requiring a total of 4 time slots to retransmit all the 4 packets. In a DNC based scheme, $A P_{1}$ and $A P_{2}$ transmit the encoded packet $c_{1} \oplus c_{2}$ and $c_{3} \oplus c_{4}$ respectively which the receivers can decode using the packet each receiver already has. Therefore a DNC based retransmission scheme requires a total of 2 time slots. JNC further improves on the retransmission gain by allowing both the APs to simultaneously retransmit $c_{1} \oplus c_{2}$ and $c_{3} \oplus c_{4}$, which results in the 2-layer encoded packet $\left(c_{1} \oplus c_{2}\right) \odot\left(c_{3} \oplus c_{4}\right)$ received at $R_{1}$ and $R_{3}$, while receiver $R_{2}$ and $R_{4}$ receive the XOR-coded packet $c_{1} \oplus c_{2}$ and $c_{3} \oplus c_{4}$ respectively, as these receivers do not fall in the interference region. Since $R_{1}$ has packet $c_{3}$ and $c_{4}$, it can use these packets to generate $c_{3} \oplus c_{4}$ and decode $\left(c_{1} \oplus c_{2}\right) \odot\left(c_{3} \oplus c_{4}\right)$ using ANC decoding, it then performs XOR decoding to retrieve packet $c_{1}$ from $c_{1} \oplus c_{2}$. Therefore in a JNC based retransmission scheme, a total of 1 time slots are needed to retransmit the lost packet. Therefore for this simple example JNC provides a retransmission gain of 4 over ARQ, and 2 over DNC.

\section{B. Non-Cooperative Collision Coding}

Each AP is only aware of the packet reception status of the receivers located within its transmission range. In our model, both the APs start the retransmission phase after transmitting $B$ packets using IEEE 802.11 based Carrier Sense Multiple Access Collision Avoidance (CSMA/CA). The retransmission process take place in 2 stages, the first stage is non-cooperative packet transmission, whereas the second stage is cooperative packet transmission. In the first stage, since the interfering AP is not aware of the packet reception status of receivers not within its transmission range, both the AP make independent 
TABLE II

ANC-CR CODING ALGORITHM, PSEUdocode

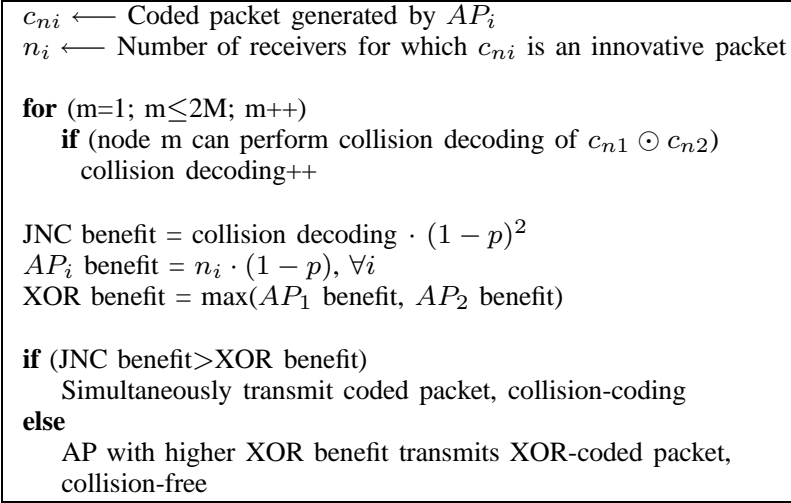

DNC coding decisions. Receivers in the non-interference region receive an XOR coded packet, whereas receivers in the interference region receive a collided XOR coded packet.

However since each of the AP make such coding algorithm decisions independently, receivers in the interference region may not necessarily benefit from such transmissions. This is because, receivers in the non-interference region only need to perform XOR decoding, whereas receivers in the interference region need to perform both ANC and XOR decoding. So while each AP can make coding decision such that the coded packet can be XOR decoded by every receiver in the multicast group of that AP, receivers in the interference region may not necessarily be able to perform ANC decoding of the collided packet from the interfering AP.

In the non-cooperative collision retransmission phase receivers in the interference region can therefore perform collision decoding opportunistically. Let $k$ represent the cardinality of the XOR-coded packet, BENEFIT coding algorithm [5] is designed such that $1 \leq k \leq N$. The probability that a receiver can opportunistically perform collision decoding is given as the product of the probability it receives a correct collided packet, and the probability that it has already opportunistically overheard the $k$ packets from the interfering AP previously, $(1-p)^{2+k}$. Therefore given the higher packet reception probability for receivers in the non-interference region, receivers in the non-interference region recover the lost packets much earlier than the receivers in the interference region. Once all the receivers in the non-interference region have correctly received the lost packets, the APs can then perform Cooperative Collision Coding.

\section{Cooperative Collision Coding}

In the Cooperative Collision phase, only the receivers in the interference region need to recover the lost packets. Because of the broadcast nature of superimposed acknowledgement, both the APs are aware of all the packet reception status of all the receivers in the interference region, and since both the APs run the same ANC-CR coding algorithm, both the APs are also aware of the coding decision the interfering AP makes. A pseudocode of the ANC-CR algorithm is given in Table II.

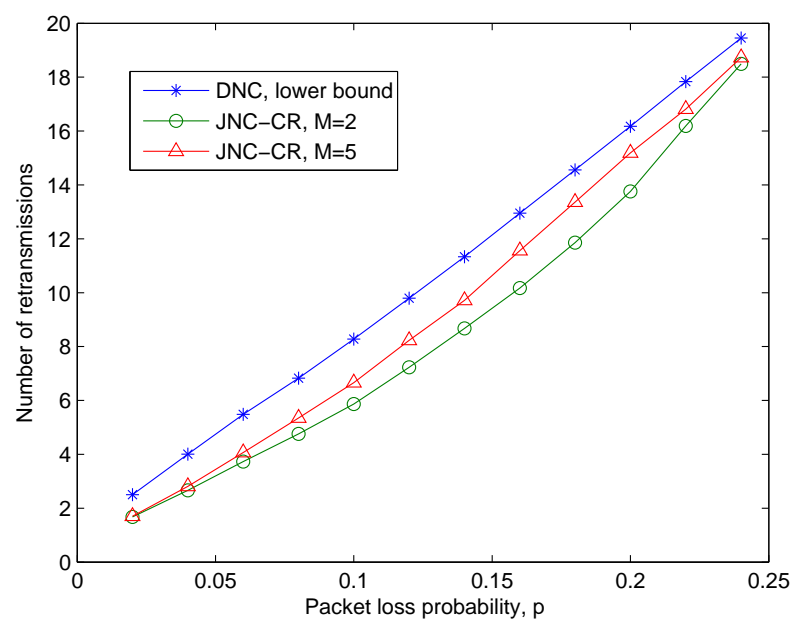

Fig. 2. Average number of retransmission under different $p$ values, for $\mathrm{N}=5$ and $\mathrm{B}=20$.

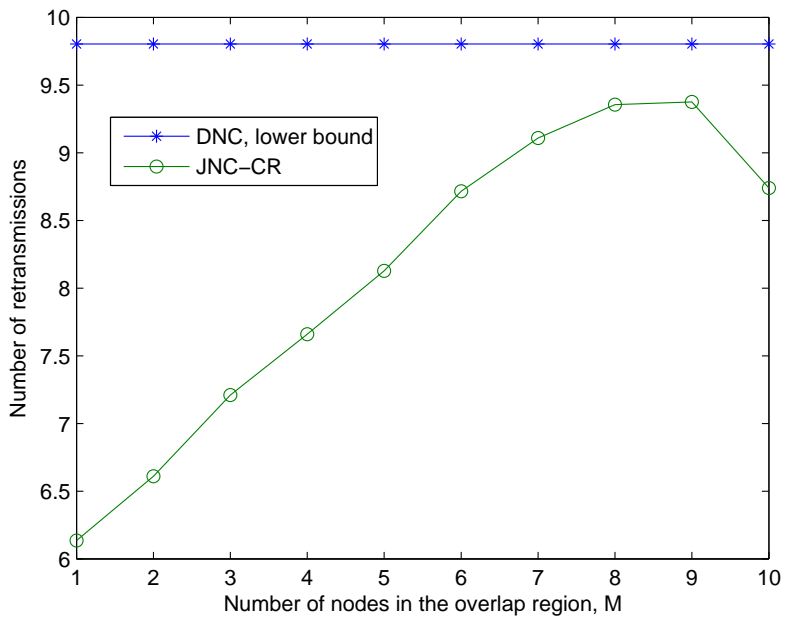

Fig. 3. Average number of retransmission under different $M$ values, for $\mathrm{N}=10, \mathrm{p}=0.1$ and $\mathrm{B}=20$.

Both the APs simultaneously run the same coding algorithm, and weigh in the benefit of simultaneously transmitting the coded packet. If the benefit of simultaneously transmitting the coded packet is greater than the benefit of transmitting either of the coded packet without collision, then both the APs transmit their coded packet simultaneously, which result in the collision of the coded packet. If however the benefit of transmitting an XOR from either AP is higher than JNC-CR, then the AP with higher transmission benefit transmit the packet. As we had shown in [9] such cooperative retransmission can be implemented without any complex handshaking or scheduling procedures.

\section{Simultation}

Packet decoding using ANC has been successfully demonstrated on a test bed in [7]. Therefore we can assume that 


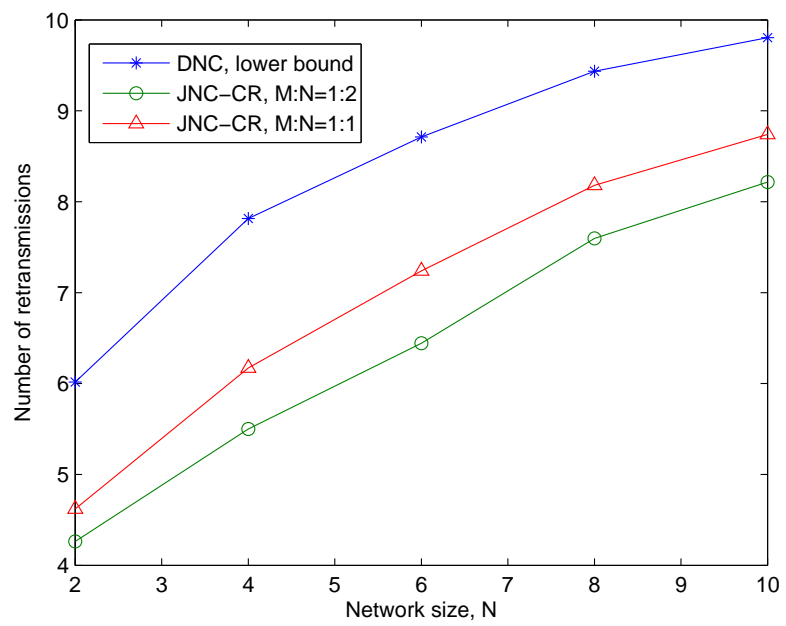

Fig. 4. Average number of retransmission under different $N$ values, for $\mathrm{p}=0.1$ and $\mathrm{B}=20$.

ANC is a practically applicable technique. For the proposed collision based cooperative retransmission, we construct a $\mathrm{C}++$ discrete-time simulator with the system model described in Section III.

Fig. 2 shows the average number of retransmission for different $p$. As $p$ increases the number of retransmission also increases for both DNC and JNC-CR, consistent with [4] [10] [15]. JNC-CR shows retransmission gain over DNC. The higher gain for decreasing $M$ in Fig. 2 and 3 comes from the cooperative collision coding stage. The probability that the receiver in the interference region will be able to perform ANC-decoding is given as $(1-p)^{2+k}$, for cooperative collision coding, $1 \leq k \leq M$. Therefore for $M=2$ more packets get ANC-coded compared to $M=5$, which improves the retransmission gain. For Fig. 3, a sudden dip in the number of retransmission occurs for $M=10$, because all the packets are then retransmitted in a cooperative collision coding, and results in a more efficient ANC coding decision.

Fig. 4 shows that the average number of retransmission increases logarithmically as the network size increases. Fig. 5 shows that the average number of retransmissions decreases logaritmically for increasing packet batch size. However using a large packet batch size will increase transmission latency. The results of Fig. 4 and 5 are consistent with [4] [10] [15]. For both these figures, JNC-CR shows better retransmission bandwidth than DNC.

\section{CONCLUSION}

In this work we have demonstrated the retransmission bandwidth gain of JNC-CR over DNC. Such a scheme can be implemented without any complex handshaking or scheduling procedure, and address the current wireless bandwidth demand, and increasing density of wireless networks in metropolitan areas. JNC-CR can also address the intra-flow interference problem in wireless multicast routing.

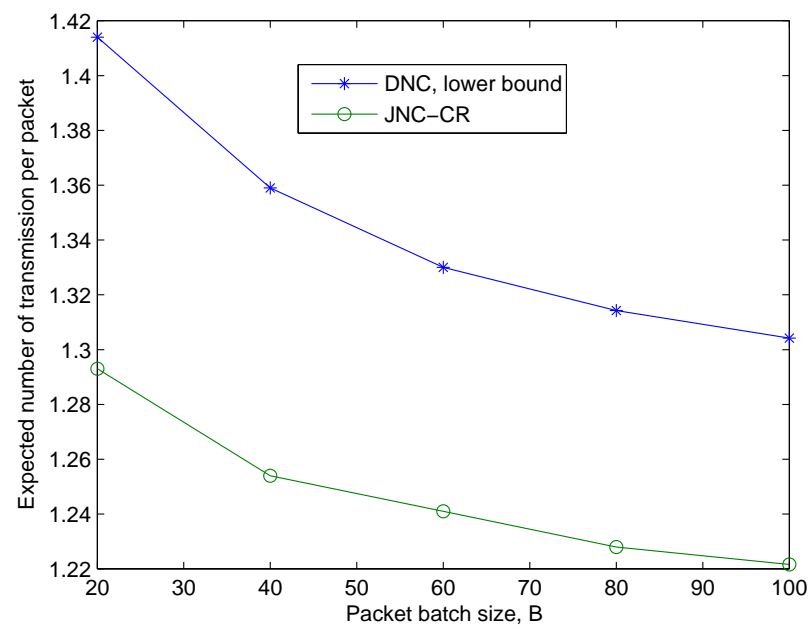

Fig. 5. Expected number of transmissions per packet for different packet batch size, for $\mathrm{p}=0.1, \mathrm{~N}=5$ and $\mathrm{M}=2$.

\section{REFERENCES}

[1] Y. Kondo, H. Yomo, S. Yamaguchi, P. Davis, R. Miura, and S. Obana, "Reliable wireless broadcast with random network coding for real-time applications," in IEEE WCNC, Budapest, Hungary, April 2009.

[2] A. Akella, G. Judd, S. Seshan, and P. Steenkiste, "Self management in chaotic wireless deployments," in ACM MobiCom, Cologne, Germany, 2005.

[3] S. S. Karande, Z. Wang, and J. G.-L.-A. Hamid R. Sadjadpour, "Multicast throughput order of network coding in wireless ad-hoc networks," IEEE Transactions on Communications, vol. 59, no. 2, pp. 497-506, February 2011.

[4] D. Nguyen, T. N. T. Tran, and B. Bose, "Wireless broadcast using network coding," IEEE Transactions on Vehicular Technology, vol. 58, no. 2, pp. 914-925, February 2009.

[5] J. Qureshi, C. H. Foh, and J. Cai, "An efficient network coding based retransmission algorithm for wireless multicast," in IEEE PIMRC, Tokyo, Japan, September 2009.

[6] S. Zhang, S. C. Liew, and P. P. Lam, "Hot topic: physical-layer network coding," in ACM MobiCom, Los Angeles, USA, September 2006.

[7] S. Katti, S. Gollakota, and D. Katabi, "Embracing wireless interference: Analog network coding," in ACM SIGCOMM, Kyoto, Japan, 2007.

[8] C. Chen and H. Xiang, "The throughput order of ad hoc networks with physical-layer network coding and analog network coding," in IEEE ICC, Beijing, China, May 2008.

[9] J. Qureshi, J. Cai, and C. H. Foh, "Cooperative retransmissions through collisions," in IEEE ICC, Kyoto, Japan, June 2011.

[10] E. Rozner, A. Padmanabha, Y. Mehta, L. Qiu, and M. Jafry, "ER: efficient retransmission scheme for wireless LANs," in ACM CoNEXT, New York, USA, December 2007.

[11] D. C. Salyers, A. Striegel, and C. Poellabauer, "Wireless reliability: Rethinking 802.11 packet loss," in IEEE WoWMoM, Newport Beach, USA, 2008

[12] C. H. Foh, J. Cai, and J. Qureshi, "Collision codes: Decoding superimposed BPSK modulated wireless transmissions," in IEEE CCNC, Las Vegas, USA, January 2010.

[13] M. Durvy, C. Fragouli, and P. Thiran, "Towards reliable broadcasting using ACKs," in IEEE ISIT, Nice, France, 2007.

[14] S. E. Rouayheb, M. Chaudhry, and A. Sprintson, "On the minimum number of transmissions in single-hop wireless coding networks," in IEEE Information Theory Workshop, California, USA, September 2007.

[15] Y. E. Sagduyu and A. Ephremides, "On network coding for stable multicast communication," in IEEE MILCOM, Florida, USA, October 2007. 